Revista Colombiana de Obstetricia y Ginecología Vol. 58 No. 2 • 2007 • (142-150)

ARtículo DE REVISIÓN

\title{
PATOGÉNESIS DE LA OSTEOPOROSIS: PAPEL DE LOS ESTRÓGENOS
}

\author{
Pathogenesis of osteoporosis: the role of estrogen \\ Oswaldo Rincón-Sierra, M.D., * Ivonne Díaz-Yamal, M.D. **, \\ Luis Ernesto Pérez-Agudelo, M.D.*** \\ Recibido: noviembre 28/06 - Revisado: febrero 7/07 - Aceptado: mayo 25/07
}

\section{RESUMEN}

Introducción: la osteoporosis es una enfermedad en la que la pérdida de fortaleza ósea conduce a fracturas por fragilidad. Las fracturas de cadera tienen alta morbilidad y mortalidad, igualmente existen resultados similares para las fracturas vertebrales. La prevalencia creciente y los tremendos costos de este desorden resaltan la importancia de desarrollar nuevos tratamientos, por ello identificar los mecanismos moleculares que controlan la masa ósea es de suma importancia.

La resorción ósea por los osteoclastos se acopla con la formación por los osteoblastos, proceso equilibrado que remodela y adapta el esqueleto. La fragilidad ósea es consecuencia de una adaptación fallida. Durante la menopausia, la remodelación del hueso pierde su equilibrio y se produce pérdida ósea.

La deficiencia de estrógenos conduce a un aumento global en producción de IL-7, en parte a través de disminución en TGF- $B$ y aumento de IGF-1, lo que lleva a activación de células T. Las células T activadas liberan IFN- $\gamma$, el cual incrementa la presentación antigénica.

\footnotetext{
* Especiualista en Endocrinología. Departamento de Medicina Interna, Hospital Militar Central. Correo electrónico: oswaldorinconsierra832@yahoo.com

** Especialista en Ginecología y Obstetricia. Jefe de la Unidad de Infertilidad y Endocrinología Reproductiva, Hospital Militar Central. Directora Científica Unidad de Fertilidad Clínica Marly (Bogotá). Correo electrónico:idiazyamal@gmail.com

*** Especialista en Ginecología y Obstetricia. Profesor asistente Universidad Militar Nueva Granada.
}

La deficiencia de estrógenos también amplifica la activación de células T y la osteoclastogénesis, regulando a la baja las vías antioxidantes. El aumento resultante en los oxidantes estimula la presentación antigénica y la producción de TNF por los osteoclastos maduros. El efecto combinado de IFN- $\gamma$ y oxidantes promueve la liberación de los factores osteoclastogénicos RANKL y TNF.

El TNF estimula la producción de RANKL y MCSF por células pluripotenciales y osteoblastos, incrementando la producción de estos últimos. TNF e IL-7 alteran la formación ósea a través de efectos represivos directos en los osteoblastos.

Palabras clave: menopausia, osteoporosis, estrógenos, patogénesis.

\section{SUMMARY}

Introduction: osteoporosis is a disorder in which loss of bone strength leads to fragility fractures. Hip fractures have very high morbidity and mortality and there are similar findings regarding vertebral fractures. This disorder's increasing prevalence and tremendous costs underscore the importance of developing new treatment. Identifying the molecular mechanisms controlling bone mass has thus become a priority task.

Bone resorption by osteoclasts is coupled to bone formation by osteoblasts, such balanced process continuously remodelling and adapting the skeleton. Bone fragility results from failed adaptation. Bone remodelling becomes unbalanced at menopause 
and results in bone loss.

Oestrogen deficiency leads to an overall increase in IL-7 production, partly through decreased TGF-B and increased IGF-1. This leads to T-cell activation. Activated T-cells release IFN- $\gamma$, thereby increasing antigen presentation. Oestrogen deficiency also amplifies T-cell activation and osteoclastogenesis by down-regulating antioxidant pathways. The resulting increase in oxidants stimulates antigen presentation and TNF production by mature osteoclasts. The combined effect of IFN- $\gamma$ and oxidants promotes RANKL and TNF osteoclastogenic factor release. TNF stimulates stem cells and RANKL and M-CSF osteoblast production, driving osteoclast formation. TNF and IL-7 blunt bone formation through direct repressive effects on osteoblasts.

Key words: menopause, osteoporosis, estrogen, pathogenesis.

\section{Definición y carga de la enfermedad}

La osteoporosis, definida, según los criterios de la OMS, como un T score menor o igual a $-2,5$, es la alteración ósea más común en el ser humano, y su característica principal es la disminución de la fortaleza ósea, lo cual conlleva a incremento en el riesgo de fracturas; la fortaleza ósea depende de dos factores fundamentales, la calidad mineral ósea y la densidad mineral ósea. ${ }^{1-4}$

Estudios en población de mujeres blancas en los Estados Unidos de Norteamérica como el National Health and Nutrition Examination Survey (NHANES) III han revelado que, de acuerdo con los criterios de la OMS, el 13-18\% (4-6 millones) de las mujeres de 50 años o mayores tienen osteoporosis de la cadera y 37-50\% (13-17 millones) tienen osteopenia. La prevalencia de la osteoporosis se incrementa con la edad avanzada siendo el $4 \%$ en mujeres de 50-59 años y alcanzando hasta el 52\% a los 80 años. ${ }^{5,6}$

Entre mujeres de 65-84 años la osteoporosis es factor fundamental en el $90 \%$ de las fracturas de columna y cadera; el riesgo durante los años siguientes de vida para una mujer blanca de 50 años de sufrir una fractura por osteoporosis es aproximadamente del 40\%. ${ }^{7,8}$

Las fracturas debidas a osteoporosis conllevan un gran impacto en cuanto a mortalidad, morbilidad, costos a los servicios de salud y deterioro en la calidad de vida; las fracturas de cadera producen un incremento en el riesgo relativo de mortalidad del 6,8 en el año siguiente a su ocurrencia y las fracturas vertebrales clínicamente evidentes un incremento del 8,64; el 25\% de mujeres requieren cuidados permanentes y el $50 \%$ pierden movilidad a largo plazo. ${ }^{9}$

En este contexto es imprescindible el conocimiento de los mecanismos implicados en la génesis de la osteoporosis con el propósito de generar estrategias preventivas y de tratamiento que permitan disminuir el gran impacto que esta enfermedad produce. Con los conocimientos actuales es claro que la osteoporosis es el resultado de la interacción de múltiples factores locales y sistémicos que actúan sobre el hueso.

\section{Aspectos básicos del metabolismo óseo}

Los condrocitos y osteoblastos se derivan de células pluripotenciales mesenquimales. Las diferentes etapas de su desarrollo están determinadas por factores de transcripción específicos como Runx2 y Sox 9; adicionalmente los adipocitos, también derivados de las células mesenquimales, pueden "diferenciarse" hacia células de la línea progenitora de osteoblastos y osteocitos; por el contrario, los osteoclastos se derivan de la línea monocito-macrófago. ${ }^{10}$

Las unidades celulares óseas (UCO) pueden formarse tanto en la superficie de los huesos trabeculares como de los corticales; la activación de los precursores de los osteoclastos se inicia por su interacción con células de la línea de los osteoblastos, pero también por células del sistema inflamatorio, particularmente los linfocitos T. Los osteoclastos inician el proceso de resorción ósea, el cual es corto y termina probablemente por apoptosis de los osteoclastos; el tiempo requerido para el proceso de formación, liderado por los osteoblastos, es más 
prolongado, generando cierto grado de debilidad durante la fase activa de remodelamiento. La osteoporosis implica una alteración en el equilibrio de estos dos procesos: un exceso de resorción, un déficit de formación o ambos mecanismos. ${ }^{11,12}$

\section{Fisiopatología}

La osteoporosis se puede producir por tres mecanismos básicos: a) Falla en la adquisición de una masa y calidad mineral ósea adecuadas durante el crecimiento; b) resorción ósea excesiva que produce disminución de la masa ósea y deterioro de la microarquitectura del hueso; c) respuesta de formación inadecuada ante la resorción ósea. ${ }^{11}$

La regulación de la formación del patrón esquelético y su desarrollo está lógicamente influenciado por determinantes genéticos: los polimorfismos de los receptores, hormonas, citoquinas, enzimas y los constituyentes de la matriz ósea hacen de la osteoporosis una enfermedad muy posiblemente poligénica.

Los estudios en gemelos indican que la predisposición genética determina hasta el $80 \%$ de la masa ósea pico, mientras el restante $20 \%$ es modulado por factores ambientales y los niveles de hormonas sexuales durante la pubertad. ${ }^{13}$

El aumento del tamaño de los huesos largos se realiza por crecimiento del hueso endocondral, mientras en los huesos planos se lleva a cabo el proceso de osificación intramembranosa. ${ }^{14}$

El déficit de calcio o las alteraciones en su vías metabólicas son mecanismos implicados en la génesis de la osteoporosis: la disminución en la ingesta de calcio, la malabsorción o el déficit de vitamina D (debajo de $30 \mathrm{ng} / \mathrm{ml}$ ) pueden conducir a un hiperparatiroidismo secundario, que lleva a pérdida acelerada de la masa ósea e incremento en la fragilidad.

Los polimorfismos en el receptor de vitamina D (VDR) se han implicado en la osteoporosis con resultados no conclusivos en los estudios realizados, por el contrario, los polimorfismos del receptor sensor de calcio, el cual es fundamental en los mecanismos contrarregulatorios para la paratohormona, no han mostrado resultados similares. ${ }^{11}$

La interacción entre los osteoblastos y los osteoclastos en el proceso de remodelación ósea es bien conocida; los osteoblastos producen RANKL, que es un ligando para el receptor activador del NF- $\kappa B$ (RANK), el cual mantiene la función de los osteoclastos y activa su diferenciación; los osteoblastos también producen osteoprotegerina (OPG) la cual inhibe la interacción RANK/RANKL.

Varios de los mecanismos implicados en el desarrollo de la osteoporosis pueden ser mediados por el aumento del RANKL, que puede incluso ser expresado en forma soluble por los linfocitos $T$ activados. La disminución en los niveles de OPG no se ha asociado consistentemente con osteoporosis, mientras que los polimorfismos de su gen sí lo han hecho con relación a fracturas osteoporóticas y disminución de la densidad mineral ósea. ${ }^{11}$

Los polimorfismos en los genes implicados en la diferenciación de los osteoblastos (Rnx2, osterix, Wnt, LRP5, BMP2) han mostrado relación con la densidad mineral ósea en estudios experimentales y pueden estar involucrados en la patogénesis de la osteoporosis; los inhibidores de la función de estos genes como SFRP y la esclerostatina también modulan la densidad mineral ósea; el déficit de esclerostatina produce la enfermedad de Van Buchem caracterizada por una densidad mineral ósea elevada. ${ }^{11}$

Es un hecho demostrado que muchas citoquinas pueden inhibir o promover la resorción o formación ósea, la prostaglandina E2, la cual se produce por las células óseas por medio de la ciclooxigenasa inducible 2, puede estimular tanto la formación como la resorción. Estudios epidemiológicos han mostrado pequeños incrementos en la DMO con el uso de antiinflamatorios no esteroideos; los polimorfismos en los genes de IL-1, IL-6, TNF- $\alpha$ y sus receptores pueden tener influencia en la masa ósea; los leucotrienos pueden estimular la resorción e inhibir la formación ósea. ${ }^{11}$

Los polimorfismos en los genes de factores de crecimiento tanto locales como sistémicos (BMP, 
IGF, TGF-B) han mostrado relación con la DMO. El óxido nítrico (NO) es un cofactor para la respuesta anabólica inducida por la carga mecánica y puede inhibir la resorción ósea al aumentar la producción de OPG. ${ }^{11}$

Los polimorfismos del gen que codifica para la cadena $\alpha 1$ del colágeno tipo I podrían explicar la disminución en la resistencia ósea independiente de la densidad mineral ósea. ${ }^{11}$

La resistencia a la leptina o su deficiencia se asocian a incrementos en la DMO, efecto atribuido a un aumento de actividad adrenérgica, los betabloqueadores han mostrados disminución del riesgo de fractura en algunos estudios epidemiológicos, resultados que han sido inconsistentes; el bloqueo de la actividad de la vía cannabinoide tipo I podría mejorar la DMO. ${ }^{11}$

\section{El papel de los estrógenos}

Las células óseas: osteoblastos, osteocitos y osteoclastos expresan receptores funcionales de estrógenos (ER). ${ }^{15,16}$ Tanto los receptores de estrógenos $\alpha$ $(\mathrm{ER} \alpha)$ como $\beta(\mathrm{ER} \beta)$ están presentes en las células óseas, aunque su distribución es diferente: ER $\alpha$ predomina en hueso cortical, mientras ERß lo hace en el hueso trabecular, sin embargo la mayoría de los efectos de los estrógenos en el hueso se realizan a través del ER $\alpha .^{17}$

Los efectos de los estrógenos se producen mediante cambios conformacionales que promueven la dimerización de receptores y unión a secuencias específicas de DNA (elementos de respuesta a estrógenos, ERE), los cuales con la unión posterior de elementos coactivadores, producen cambios en la maquinaria de transcripción del DNA. ${ }^{18}$

Existen mecanismos adicionales por medio de los cuales los estrógenos pueden modificar la expresión de genes implicados en el proceso de remodelación ósea: unión de ER al NF- $\kappa \beta$ impidiendo su unión al DNA y la expresión de IL-6 y disminuyendo la actividad caseinquinasa 2 (CK2), lo cual reduce la fosforilación de Egr-1 incrementando la afinidad de esta por el activador transcripcional Sp-1 y limitando la disponibilidad de este a nivel nuclear, y, como consecuencia reduciendo la expresión del gen MCSF. ${ }^{19}$

También se ha demostrado la capacidad de los estrógenos de suprimir la actividad quinasa de JNK (Quinasa Jun - N - Terminal), contribuyendo a una menor expresión de TNF y reduciendo la sensibilidad de los osteoclastos a la actividad RANK/RANKL. ${ }^{20}$

La acción antiapoptótica de los estrógenos sobre los osteoblastos y proapoptótica sobre los osteoclastos se debe a su capacidad para incrementar la fosforilación de las quinasas citoplasmáticas ERK1 y 2 y suprimir la actividad JNK. ${ }^{21}$

El déficit de estrógenos produce incremento en el número de unidades de remodelación ósea activadas por unidad de tiempo, interactuando con células del sistema inmune, primordialmente por aumento de la formación de osteoclastos y reducción de su apoptosis. ${ }^{22}$

Aunque existe un incremento en la respuesta de formación ósea, esta es inadecuada para el grado de resorción, ya que también se evidencia un mayor grado de apoptosis de los osteoblastos. ${ }^{23}$

La producción incrementada de citoquinas inflamatorias como IL-7 y TNF limita la actividad de los osteoblastos maduros..$^{24} \mathrm{La}$ terapia de reemplazo con estrógenos produce incremento de la apoptosis de los osteoclastos y disminución de la apoptosis de los osteoblastos. ${ }^{25,26}$

Las condiciones basales mínimas para la formación de los osteoclastos son la presencia de RANKL y M-CSF, que son producidos por los osteoblastos maduros, las células precursoras de la medula ósea y las células $\mathrm{T}$ activadas.

Como ya se mencionó, el sistema RANKL/ RANK permite la diferenciación y actividad de los osteoclastos, mientras la OPG, como su nombre lo indica, actúa protegiendo el hueso. El M-CSF induce la proliferación de los precursores tempranos de los osteoclastos, su diferenciación y fusión a osteoclastos maduros e incrementa su supervivencia. ${ }^{27}$

La deficiencia de estrógenos induce la producción 
de citoquinas proinflamatorias como TNF, la cual estimula la osteoclastogénesis directamente y por medio de la producción de RANKL en las células precursoras de la medula ósea. Adicionalmente la respuesta de los osteoclastos se incrementa y se produce regulación al alta del RANK. Otro efecto que favorece el incremento neto de la resorción ósea es la disminución de la osteoblastogénesis. ${ }^{28-31}$ El estímulo de la osteoclastogénesis por TNF está mediado por interacciones a nivel del NF- $\kappa \beta$ y señales AP1. ${ }^{32}$

Los estudios experimentales de animales transgénicos insensibles a TNF (déficit de receptor o un receptor soluble) han demostrado que estos animales se encuentran protegidos contra la pérdida ósea inducida por la ooforectomía. Existe evidencia del aumento de TNF en mujeres posmenopáusicas. ${ }^{33-35}$

La producción de estas cantidades incrementadas de TNF se debe a las células T primordialmente y el tratamiento con estrógenos induce disminución del grupo de células responsables de su producción. ${ }^{29,36}$

Las células T activadas también producen IFN$\alpha$ e IFN- $\gamma$, los cuales tienen efecto antiosteoclastogénico al suprimir la actividad NF-KB y JNK en determinadas circunstancias; durante estados inflamatorios y durante la deficiencia de estrógenos predomina la producción de un perfil de citoquinas que favorece la resorción ósea. ${ }^{37-39}$

Se ha demostrado que el incremento en la producción de TNF inducido por la ooforectomía está principalmente mediado por un aumento de las células T productoras de TNF. ${ }^{39}$ La regulación de la activación y proliferación de estas células se lleva a cabo a nivel del timo, la medula ósea y los órganos linfoides periféricos. ${ }^{40}$

La deficiencia de estrógenos produce regulación al alta de las moléculas del CMH clase II, proceso que es mediado por la expresión del gen transactivador de clase II (CIITA) y que es inducido por IFN- $\gamma$, hecho demostrado por la falta de respuesta de pérdida ósea inducida por ooforectomía en los ratones que carecen del receptor de IFN- $\gamma$. El IFN- $\gamma$ también tiene efectos osteoclastogénicos mediados por la vía RANKL. ${ }^{41}$

El incremento en la producción de IFN- $\gamma$ en la deficiencia de estrógenos está mediado por la disminución del TGF-B, ya que los estrógenos son inductores de su producción por medio de acciones genómicas en el promotor del TGF-B. ${ }^{42}$

El TGF- $B$ es un potente inmunosupresor que inhibe la proliferación y activación de las células T y su producción de citoquinas inflamatorias como IFN- $\gamma{ }^{43}$ Un dato muy importante en los modelos de ratones transgénicos con bloqueo de la acción del TGF- $\beta$ es su resistencia a los efectos protectores del hueso mediados por estrógenos, asociado a una falla en la supresión de IFN- $\gamma$, con el consecuente incremento en la activación y proliferación de las células T, lo cual conlleva a niveles incrementados de TNF. ${ }^{44}$

Otro importante mecanismo de modulación en la remodelación ósea mediada por estrógenos implica la intervención de la IL-7, la cual tiene un efecto proinflamatorio y estimulador de la resorción ósea. ${ }^{45}$ Los mecanismos por los cuales la IL-7 favorece la resorción ósea implican actividad del RANKL y el TNF. ${ }^{46}$

En la ooforectomía experimental se ha demostrado incremento en los niveles de IL-7, y la falta de expresión de esta citoquina protege de la pérdida ósea inducida por ooforectomía. ${ }^{47}$

Los mecanismos proinflamatorios de la IL-7 incluyen la expansión de las células $\mathrm{T}$ al disminuir su tolerancia antigénica e incrementan la presentación antigénica mediada por IFN- $\gamma$, disminuyendo los niveles de TGF-B. La IL-7 tiene efectos estimulatorios tanto en células $\mathrm{T}$ y $\mathrm{B}$, estas últimas podrían tener un papel en la génesis de la osteoporosis mediado por actividad RANKL. ${ }^{48-50}$

Tanto los andrógenos como los estrógenos son potentes supresores de la actividad tímica, pues promueven la apoptosis y la detención de la diferenciación de células del sistema inmune; la castración induce la proliferación de células tímicas y su liberación a la periferia. ${ }^{51,52}$

El timo aporta aproximadamente el 50\% del 
incremento en las células $\mathrm{T}$ inducido por ooforectomía y la timectomía reduce la pérdida ósea en un $50 \%$. Estos hallazgos deben ser confirmados en humanos. ${ }^{53}$

En modelos de osteoporosis posmenopáusica se ha demostrado que la deficiencia de estrógenos induce la producción de oxidantes, radicales libres de oxígeno, y disminuye la producción de antioxidantes; el suplemento con agentes antioxidantes como la $\mathrm{N}$-aceltilcisteína previene la pérdida ósea en un ambiente bajo en estrógenos. ${ }^{54,55}$

Los radicales libres tienen la capacidad de promover la generación y diferenciación de los osteoclastos, mecanismo mediado por la inhibición reversible de fosfatasas y la expresión de tioredoxina, la cual promueve la generación de los osteoclastos. ${ }^{56,57}$

Los estrógenos promueven la producción de glutatión peroxidasa en los osteoclastos, enzima que degrada el peróxido de hidrógeno, un poderoso oxidante ${ }^{58} \mathrm{La}$ presencia de donadores de óxido nítrico previene la pérdida ósea inducida por ooforectomía; por el contrario, los inhibidores de la óxido-nítricosintetasa hacen inefectiva la acción protectora del hueso mediada por estrógenos. Estudios en humanos han demostrado que la nitroglicerina protege de fracturas por osteoporosis en mujeres posmenopáusicas. ${ }^{59-61}$

La generación de TNF en los osteoclastos es inducida por radicales libres, un proceso con gran similitud a los estados de deficiencia estrogénica. Los radicales libres son estimuladores potentes de la actividad de las células inflamatorias, induciendo la actividad de las células presentadoras de antígenos, la expresión de moléculas coestimulatorias, y favoreciendo la actividad de las células T. ${ }^{62}$

En conclusión, el déficit de estrógenos induce la acción orquestada de hormonas y citoquinas que se integran para alterar el proceso de remodelación ósea. Los eventos iniciales pueden ser la disminución del TGF-B y el aumento de IGF-1, lo cual conduce a niveles incrementados de IL-7. Esta citoquina actúa sobre el timo, hueso y bazo, conduciendo a la activación de las células T.

Las células T activadas liberan IFN- $\gamma$, que induce la activación del factor de transcripción CIITA, el cual produce aumento de la expresión de las moléculas del CMH II, conduciendo al incremento de la presentación antigénica por las células como los macrófagos.

La deficiencia de estrógenos produce disminución de los antioxidantes, la cual aumenta el nivel de radicales libres de oxígeno, que amplifican la activación de las células T, la presentación antigénica, la osteoclastogénesis y la producción de TNF por los osteoclastos.

Los efectos combinados de IFN- $\gamma$ y los radicales libres de oxígeno incrementan la presentación antigénica, la activación de las células T y promueven la liberación de factores osteoclastogénicos como RANKL y TNF.

El TNF al incrementar los niveles de IL-1 estimula la producción de RANKL y M-CSF en las células estromales y los osteoblastos, conduciendo a la formación de osteoclastos. Adicionalmente el TNF y la IL-7 tienen efectos supresores sobre los osteoblastos.

\section{REFERENCIAS}

1. Kanis JA. Assessment of fracture risk and its application to screening for postmenopausal osteoporosis: synopsis of a WHO report. WHO Study Group. Osteoporos Int 1994;4:368-81.

2. NIH Consensus Development Panel on Osteoporosis Prevention, Diagnosis, and Therapy. Osteoporosis prevention, diagnosis, and therapy. JAMA 2001;285:785-95.

3. Hodgson SF, Watts NB, Bilezikian JP, Clarke BL, Gray TK, Harris DW, et al. American Association of Clinical Endocrinologists medical guidelines for clinical practice for the prevention and treatment of postmenopausal osteoporosis: 2001 edition, with selected updates for 2003. Endocr Pract 2003;9:544-64.

4. North American Menopause Society. Management of osteoporosis in postmenopausal women: 2006 position statement of The North American Menopause Society. Menopause 2006;13:340-67.

5. Looker AC, Wahner HW, Dunn WL, Calvo MS, Harris TB, Heyse SP, et al. Updated data on proximal femur 
bone mineral levels of US adults. Osteoporos Int 1998;8:468-89.

6. Looker AC, Orwoll ES, Johnston CC, Lindsay RL, Wahner HW, Dunn WL, et al. Prevalence of low femoral bone density in older U.S. adults from NHANES III. J Bone Miner Res 1997;12:1761-8.

7. Melton LJ, Thamer M, Ray NF, Chan JK, Chesnut $\mathrm{CH}$, Einhorn TA, et al. Fractures attributable to osteoporosis: report from the National Osteoporosis Foundation. J Bone Miner Res 1997;12:16-23.

8. Johnell O, Kanis J. Epidemiology of osteoporotic fractures. Osteoporos Int 2005;16 Suppl 2:S3-7.

9. Cauley JA, Thompson DE, Ensrud KC, Scott JC, Black D. Risk of mortality following clinical fractures. Osteoporos Int 2000;11:556-61.

10. Russell GR, Espina B, Hulley P. Bone biology and the pathogenesis of osteoporosis. Curr Opin Rheumatol 2006;18 Suppl 1:S3-10.

11. Raisz LG. Pathogenesis of osteoporosis: concepts, conflicts, and prospects. J Clin Invest 2005;115:331825.

12. Riggs BL, Khosla S, Melton LJ. Sex steroids and the construction and conservation of the adult skeleton. Endocr Rev 2002;23:279-302.

13. Davies JH, Evans BA, Gregory JW. Bone mass acquisition in healthy children. Arch Dis Child 2005;90:373-8.

14. Kronenberg HM. Developmental regulation of the growth plate. Nature 2003;423:332-6.

15. Komm BS, Terpening CM, Benz DJ, Graeme KA, Gallegos A, Korc M, et al. Estrogen binding, receptor mRNA, and biologic response in osteoblast-like osteosarcoma cells. Science 1988;241:81-4.

16. Tomkinson A, Gevers EF, Wit JM, Reeve J, Noble BS. The role of estrogen in the control of rat osteocyte apoptosis. J Bone Miner Res 1998;13:1243-50.

17. Hall JM, McDonnell DP. The estrogen receptor beta-isoform (ERbeta) of the human estrogen receptor modulates ERalpha transcriptional activity and is a key regulator of the cellular response to estrogens and antiestrogens. Endocrinology 1999; 140:5566-78.

18. Deroo BJ, Korach KS. Estrogen receptors and human disease. J Clin Invest 2006;116:561-70.

19. Srivastava S, Weitzmann MN, Kimble RB, Rizzo M, Zahner M, Milbrandt J, et al. Estrogen blocks M-CSF gene expression and osteoclast formation by regulating phosphorylation of Egr-1 and its interaction with Sp-1. J Clin Invest 1998;102:1850-9.

20. Srivastava S, Weitzmann MN, Cenci S, Ross FP, Adler S, Pacifici R. Estrogen decreases TNF gene expression by blocking JNK activity and the resulting production of C-Jun and JunD. J Clin Invest 1999;104:503-13.

21. Kousteni S, Han L, Chen JR, Almeida M, Plotkin LI, Bellido T, et al. Kinase-mediated regulation of common transcription factors accounts for the bone-protective effects of sex steroids. J Clin Invest 2003;111:165164.

22. Weitzmann MN, Pacifici R. The role of T lymphocytes in bone metabolism. Immunol Rev 2005;208:154-68.

23. Kousteni S, Bellido T, Plotkin LI, O'Brien CA, Bodenner DL, Han L, et al. Nongenotropic, sexnonspecific signaling through the estrogen or androgen receptors: dissociation from transcriptional activity. Cell 2001;104:719-30.

24. Weitzmann MN, Roggia C, Toraldo G, Weitzmann L, Pacifici R. Increased production of IL-7 uncouples bone formation from bone resorption during estrogen deficiency. J Clin Invest 2002;110:1643-50.

25. Eriksen EF, Langdahl B, Vesterby A, Rungby J, Kassem M. Hormone replacement therapy prevents osteoclastic hyperactivity: a histomorphometric study in early postmenopausal women. J Bone Miner Res 1999;14:1217-21.

26. Manolagas SC. Birth and death of bone cells: basic regulatory mechanisms and implications for the pathogenesis and treatment of osteoporosis. Endocr Rev 2000;21:115-37.

27. Khosla S. Minireview: the OPG/RANKL/RANK system. Endocrinology 2001;142:5050-5.

28. Hofbauer LC, Lacey DL, Dunstan CR, Spelsberg TC, Riggs BL, Khosla S. Interleukin-1beta and tumor necrosis factor-alpha, but not interleukin-6, stimulate osteoprotegerin ligand gene expression in human osteoblastic cells. Bone 1999;25:255-9.

29. Cenci S, Weitzmann MN, Roggia C, Namba N, Novack D, Woodring J, et al. Estrogen deficiency induces bone loss by enhancing T-cell production of TNF-alpha. J Clin Invest 2000;106:1229-37.

30. Zhang YH, Heulsmann A, Tondravi MM, Mukherjee A, Abu-Amer Y. Tumor necrosis factor-alpha (TNF) stimulates RANKL-induced osteoclastogenesis via coupling of TNF type 1 receptor and RANK signaling pathways. J Biol Chem 2001;276:563-8.

31. Nanes MS. Tumor necrosis factor-alpha: molecular 
and cellular mechanisms in skeletal pathology. Gene 2003;321:1-15.

32. Lam J, Takeshita S, Barker JE, Kanagawa O, Ross FP, Teitelbaum SL. TNF-alpha induces osteoclastogenesis by direct stimulation of macrophages exposed to permissive levels of RANK ligand. J Clin Invest 2000;106:1481-8.

33. Weitzmann MN, Pacifici R. Estrogen deficiency and bone loss: an inflammatory tale. J Clin Invest 2006;116:1186-94.

34. Ammann P, Rizzoli R, Bonjour JP, Bourrin S, Meyer JM, Vassalli P, et al. Transgenic mice expressing soluble tumor necrosis factor-receptor are protected against bone loss caused by estrogen deficiency. J Clin Invest 1997;99:1699-703.

35. Ralston SH, Russell RG, Gowen M. Estrogen inhibits release of tumor necrosis factor from peripheral blood mononuclear cells in postmenopausal women. J Bone Miner Res 1990;5:983-8.

36. Abrahamsen B, Bendtzen K, Beck-Nielsen H. Cytokines and T-lymphocyte subsets in healthy post-menopausal women: estrogen retards bone loss without affecting the release of IL-1 or IL-1ra. Bone 1997;20:251-8.

37. Takayanagi H, Ogasawara K, Hida S, Chiba T, Murata S, Sato K, et al. T-cell-mediated regulation of osteoclastogenesis by signalling cross-talk between RANKL and IFN-gamma. Nature 2000;408:600-5.

38. Kong YY, Feige U, Sarosi I, Bolon B, Tafuri A, Morony S, et al. Activated T cells regulate bone loss and joint destruction in adjuvant arthritis through osteoprotegerin ligand. Nature 1999;402:304-9.

39. Cenci S, Toraldo G, Weitzmann MN, Roggia C, Gao Y, Qian WP, et al. Estrogen deficiency induces bone loss by increasing $\mathrm{T}$ cell proliferation and lifespan through IFN-gamma-induced class II transactivator. Proc Natl Acad Sci U S A 2003;100:10405-10.

40. Roggia C, Gao Y, Cenci S, Weitzmann MN, Toraldo G, Isaia G, et al. Up-regulation of TNF-producing T cells in the bone marrow: a key mechanism by which estrogen deficiency induces bone loss in vivo. Proc Natl Acad Sci U S A 2001;98:13960-5.

41. Kotake S, Nanke Y, Mogi M, Kawamoto M, Furuya $\mathrm{T}$, Yago $\mathrm{T}$, et al. IFN-gamma-producing human $\mathrm{T}$ cells directly induce osteoclastogenesis from human monocytes via the expression of RANKL. Eur J Immunol 2005;35:3353-63.

42. Yang NN, Venugopalan M, Hardikar S, Glasebrook
A. Identification of an estrogen response element activated by metabolites of 17 beta-estradiol and raloxifene. Science 1996;273:1222-5.

43. Gao Y, Qian WP, Dark K, Toraldo G, Lin AS, Guldberg RE, et al. Estrogen prevents bone loss through transforming growth factor beta signaling in T cells. Proc Natl Acad Sci U S A 2004;101:16618-23.

44. Miyaura C, Onoe Y, Inada M, Maki K, Ikuta K, Ito $\mathrm{M}$, et al. Increased B-lymphopoiesis by interleukin 7 induces bone loss in mice with intact ovarian function: similarity to estrogen deficiency. Proc Natl Acad Sci U S A 1997;94:9360-5.

45. Valenzona HO, Pointer R, Ceredig R, Osmond DG. Prelymphomatous $\mathrm{B}$ cell hyperplasia in the bone marrow of interleukin-7 transgenic mice: precursor B cell dynamics, microenvironmental organization and osteolysis. Exp Hematol 1996;24:1521-9.

46. Toraldo G, Roggia C, Qian WP, Pacifici R, Weitzmann MN. IL-7 induces bone loss in vivo by induction of receptor activator of nuclear factor kappa $\mathrm{B}$ ligand and tumor necrosis factor alpha from T cells. Proc Natl Acad Sci U S A 2003;100:125-30.

47. Lee SK, Kalinowski JF, Jacquin C, Adams DJ, Gronowicz G, Lorenzo JA. Interleukin-7 influences osteoclast function in vivo but is not a critical factor in ovariectomy-induced bone loss. J Bone Miner Res 2006;21:695-702.

48. Huang M, Sharma S, Zhu LX, Keane MP, Luo J, Zhang L, et al. IL-7 inhibits fibroblast TGF-beta production and signaling in pulmonary fibrosis. J Clin Invest 2002;109:931-7.

49. Dubinett SM, Huang M, Dhanani S, Economou JS, Wang J, Lee P, et al. Down-regulation of murine fibrosarcoma transforming growth factor-beta 1 expression by interleukin 7. J Natl Cancer Inst 1995;87:593-7.

50. Sato T, Shibata T, Ikeda K, Watanabe K. Generation of bone-resorbing osteoclasts from B220+ cells: its role in accelerated osteoclastogenesis due to estrogen deficiency. J Bone Miner Res 2001;16:2215-21.

51. Okasha SA, Ryu S, Do Y, McKallip RJ, Nagarkatti M, Nagarkatti PS. Evidence for estradiol-induced apoptosis and dysregulated $\mathrm{T}$ cell maturation in the thymus. Toxicology 2001;163:49-62.

52. Utsuyama M, Hirokawa K. Hypertrophy of the thymus and restoration of immune functions in mice and rats by gonadectomy. Mech Ageing Dev 1989;47:175-85. 
53. Ryan MR, Shepherd R, Leavey JK, Gao Y, Grassi F, Schnell FJ, et al. An IL-7-dependent rebound in thymic $\mathrm{T}$ cell output contributes to the bone loss induced by estrogen deficiency. Proc Natl Acad Sci U S A 2005;102:16735-40.

54. Muthusami S, Ramachandran I, Muthusamy B, Vasudevan G, Prabhu V, Subramaniam V, et al. Ovariectomy induces oxidative stress and impairs bone antioxidant system in adult rats. Clin Chim Acta 2005;360:81-6.

55. Lean JM, Davies JT, Fuller K, Jagger CJ, Kirstein B, Partington GA, et al. A crucial role for thiol antioxidants in estrogen-deficiency bone loss. J Clin Invest 2003;112:915-23.

56. Reth M. Hydrogen peroxide as second messenger in lymphocyte activation. Nat Immunol 2002;3:1129-34.

57. Lean J, Kirstein B, Urry Z, Chambers T, Fuller K. Thioredoxin-1 mediates osteoclast stimulation by reactive oxygen species. Biochem Biophys Res
Commun 2004;321:845-50.

58. Lean JM, Jagger CJ, Kirstein B, Fuller K, Chambers TJ. Hydrogen peroxide is essential for estrogen-deficiency bone loss and osteoclast formation. Endocrinology 2005;146:728-35.

59. Wimalawansa SJ, De Marco G, Gangula P, Yallampalli C. Nitric oxide donor alleviates ovariectomy-induced bone loss. Bone 1996;18:301-4.

60. Hao YJ, Tang Y, Chen FB, Pei FX. Different doses of nitric oxide donor prevent osteoporosis in ovariectomized rats. Clin Orthop Relat Res 2005;226-31.

61. Jamal SA, Cummings SR, Hawker GA. Isosorbide mononitrate increases bone formation and decreases bone resorption in postmenopausal women: a randomized trial. J Bone Miner Res 2004;19:1512-7.

62. Jagger CJ, Lean JM, Davies JT, Chambers TJ. Tumor necrosis factor-alpha mediates osteopenia caused by depletion of antioxidants. Endocrinology 2005;146:113-8. 